\title{
Fast Fragmentation During Surface-Induced Dissociation: An Examination of Peptide Size and Structure
}

\author{
George L. Barnes ${ }^{\mathrm{a}, *}$, Amanda Shlaferman ${ }^{\mathrm{a}}$, Monica Strain ${ }^{\mathrm{a}}$ \\ ${ }^{a}$ Department of Chemistry and Biochemistry \\ Siena College \\ 515 Loudon Road \\ Loudonville, NY 12211
}

\begin{abstract}
We present the results of direct dynamics simulations of surface-induced dissociation for protonated versions of $\mathrm{A}_{\mathrm{n}} \mathrm{K}, \mathrm{KA}_{\mathrm{n}}\left(\mathrm{n}=1,3\right.$, and 5), $\mathrm{AcA}_{7} \mathrm{~K}$, and $\mathrm{AcKA}_{7}$ for collisions with a fluorinated self-assembled monolayer surface. We focus on elucidating fast fragmentation events, which takes place in coincidence with the collision event. Such events generate a large number of products, and hence, are not easily understood through chemical intuition. Our simulations show distinct differences between the $\mathrm{A}_{\mathrm{n}} \mathrm{K} / \mathrm{AcA}_{7} \mathrm{~K}$ and $\mathrm{KA}_{\mathrm{n}} / \mathrm{AcKA}_{7}$ series of peptides, with the former being more reactive, and the latter more selective. Backbone rearrangements and sidechain fragmentation are also seen.
\end{abstract}

Keywords: Tandem Mass Spectrometry, Direct Dynamics Simulations, Surface-Induced Dissociation, Fast Fragmentation Events, Shattering

\section{Introduction}

Surface-induced dissociation (SID) is a well-studied analytical technique that has frequently been used to study the fragmentation of biological ions [1-3]. Surfaces such as diamond as well as organic self-assembled monolayer (SAM) surfaces are commonly used in this technique, and protonated peptides have been a particular focus of SID studies [4-6]. During SID, an ion with a known mass to charge ratio is imparted a precise distribution of translational energy and directed towards a surface with a given incidence angle. The resulting collision allows for translational to internal energy transfer with fragmentation occurring when a sufficient amount of energy is deposited into individual bonds. Hence, fragmentation can take a significant amount of time as intramolecular vibrational relaxation (IVR) redistributes the transferred energy throughout the molecule. However, it has also been seen computationally, as well as experimentally, that fragmentation can occur in coincidence with the collision event. Such fragmentation events have been termed "shattering." [4, 7-15]

Shattering is known to produce an incredibly large number of different reactive products within experiments

\footnotetext{
${ }^{*}$ Corresponding author

Email address: gbarnes@siena. edu (George L. Barnes)
}

[2] and simulations [15] of protonated peptides. Unlike statistical fragmentation of peptide ions, in which the IVR process is complete and fragmentation is often driven by proton motion (i.e. consistent with the mobile proton model $[16,17])$, it is common for shattering fragmentation to occur in a charge remote fashion. One of the incredibly nice features of proton driven fragmentation is the intuitive picture that is provided, namely that a proton is mobilized and migrates to a thermodynamically less stable site, which in turn weakens a covalent bond and makes it more likely to cleave. $[16,17]$ There is no equivalent intuitive picture available for shattering fragmentation, which is in part due to the somewhat overwhelming number of different fragmentation products possible.

There has been a significant computational focus on "shattering" in gly $_{n}-\mathrm{H}^{+}+$surface systems [11, 13-15] with $n=1,2$ and 8 . The present work provides greater insight into fast fragmentation dynamics by presenting a direct dynamics study of a complementary series of protonated peptides, namely $\mathrm{A}_{\mathrm{n}} \mathrm{K}$ and $\mathrm{KA}_{\mathrm{n}}(\mathrm{n}=1,3,5)$ as well as $\mathrm{AcA}_{7} \mathrm{~K}$ and $\mathrm{AcKA}_{7}$ colliding with a fluorinated SAM (FSAM) surface. In this context, "fast" refers to fragmentation events that are initiated within $300 \mathrm{fs}$ of impact and velocity reversal from the surface. Following this initiation, both secondary rearrangements and fragmentation events can also take place on a picosecond time 
scale. As a whole, we will refer to this type of fragmentation as a Fast Fragmentation Event (FFE). $\mathrm{AcA}_{7} \mathrm{~K}$ and $\mathrm{AcKA}_{7}$ were the subject of a previous work focused on soft-landing [18], but are also clearly complementary to the present study. This choice of protonated peptides probes very small dipeptides up to octapeptides, and allows for a limited examination of globular vs alpha-helical secondary structure while also increasing the chemical diversity of systems explored by direct dynamics. In addition, we seek to provide insight into the types of bonds that are most likely to be cleaved during these sub-picosecond events rather than focusing on individual products for a specific system. In order to accomplish this, we will identify the final product ion based on the bond cleavage sites as defined using the established nomenclature for protonated peptide ion products [19]. Moreover, we seek to determine if there is either a size or structural correlation with the types of bonds cleaved.

\section{Method}

Below we describe our method for obtaining the initial structures for each species as well as for performing the direct dynamics simulations. The work can also be viewed as an extension of that of Frederickson et al. [18], which focused on soft landing while this work focuses on picosecond and sub-picosecond fragmentation.

\subsection{Initial Structures}

Structures are needed for eight different species, namely lysine protonated versions of $A_{n} K$ and $K A_{n}$ with $n=1$, 3 , and 5 as well as $\mathrm{AcA}_{7} \mathrm{~K}$ and $\mathrm{AcKA}_{7}$. The latter two structures are taken from Frederickson et al. As discussed in Frederickson et al., Jarrold and co-workers found that $\mathrm{AcA}_{7} \mathrm{~K}$ is the shortest, stable alpha-helical peptide known, while $\mathrm{AcKA}_{7}$ has a globular form [20]. From the previous work of Barnes and co-workers[21], it is known that in simulations of similar systems, placing the excess proton on the lysine group yields the lowest energy structures. In this work, we were able to form an alpha-helical structure for $\mathrm{A}_{5} \mathrm{~K}$ that was stable for our short-time, gas phase simulations. All other structures are globular. The $\mathrm{A}_{5} \mathrm{~K}$ structure was obtained via Avogadro [22] and then optimized using the RM1 semi-empirical method [23]. RM1 was selected since it was used in the soft-landing work of Frederickson et al. as well as numerous other direct dynamics simulations of protonated peptides [21, 24-29].

The structure for all other species were obtained by obtaining an initial structure via Avogadro and reoptimizing it via GROMACS [30] with the OPLS force field fol- lowed by a 100 ps equilibration at $300 \mathrm{~K}$. A simulated annealing procedure was then applied using 20 heat-cool cycles in which the temperature was ramped to $1000 \mathrm{~K}$ over $100 \mathrm{ps}$ and then cooled to $0 \mathrm{~K}$ over an additional $100 \mathrm{ps}$. All of the $0 \mathrm{~K}$ structures were optimized at the RM1 level as implemented in Mopac2016 [31]. The lowest energy conformation was selected from these structures as the initial starting point for the direct dynamics simulations. All structures are shown in Appendix A. These species show a diverse range of conformations, and the $\mathrm{A}_{n} \mathrm{~K}$ and $\mathrm{KA}_{n}$ series have distinct differences. AK and KA both exhibit hydrogen bonding between the protonated $\mathrm{N}$ of the sidechain and the N-terminus. However, the nitrogen within the lysine group of KA also shows a close proximity to the Cterminus and has additional stabilization. This trend continues to $\mathrm{A}_{3} \mathrm{~K}$ and $\mathrm{KA}_{3}$. The nitrogen on the lysine group in $\mathrm{A}_{3} \mathrm{~K}$ is hydrogen bound to a carbonyl along the backbone, whereas $\mathrm{KA}_{3}$ retains the sidechain to $\mathrm{N}$-terminus hydrogen bond as well as being in close proximity to the C-terminus. $\mathrm{A}_{5} \mathrm{~K}$ and $\mathrm{AcA}_{7} \mathrm{~K}$ are both alpha helical, and hence, are more elongated whereas $\mathrm{KA}_{5}$ and $\mathrm{AcKA}_{7}$ have formed a "pocket" of carbonyl groups that are associated with the side chain nitrogen group. The relative energy of all structures is given in the supplemental information.

\subsection{Direct Dynamics Simulations}

Our approach to performing direct dynamics simulations of collision systems relevant to mass spectrometry has recently been detailed in three review articles [3234], and hence, we shall only provide an outline of the approach here. Several of the original works on the specific topic of protonated peptides colliding with organic self-assembled monolayers [11, 35-37] would also provide additional technical detail.

We begin by writing the potential energy as a sum of three components, namely

$$
V=V_{\text {peptide }}+V_{S A M}+V_{\text {peptide-SAM }}
$$

where $V_{S A M}$ is the intramolecular, molecular mechanical (MM) force field for FSAMs and $V_{\text {peptide-SAM }}$ is the most recent $\mathrm{MM}$ force field for the interaction between a protonated peptide and the SAM surface. This force field, developed by Hase and co-workers, is specifically designed for modeling soft landing [38, 39]. However, it also performs well at higher collision energies, such as those considered here. The peptide potential, $V_{\text {peptide }}$, is treated using the RM1 semi-empirical method for the reasons described in the previous section.

The peptide is initially given a random orientation and placed $40 \AA$ above a 9x9 octanethiol FSAM surface. Ini- 
tial positions and relative velocities were randomly selected for both the surface and the peptide using a velocity re-scaling routine such that each was given an initial temperature of $300 \mathrm{~K}$. Separate MD simulations were performed for the peptide and the surface with velocity re-scaling occurring every 30 time steps. This approach was successfully used by Frederickson et al[18]. Lastly, a collision energy of $100 \mathrm{eV}$ with a normal incidence angle was imparted to the peptide as it is sufficiently high for a significant number of both fragmentation and FFEs to occur. One thousand trajectories were calculated for each species.

Hamilton's equations of motion were integrated using a $6^{\text {th }}$ order sympletic integration scheme [40], making use of a one femtosecond step size with output written every 50 femtoseconds. Trajectories were stopped after five picoseconds of simulation time. Conservation of energy was continually checked during the simulations. If a large jump in energy conservation was seen for any given step, it was rejected and the time step was re-integrated using five 0.2 fs time steps. Assuming energy was well conserved following this, the normal time step was reinstated. This precaution was important to implement as a fairly large number of trajectories $(\sim 5 \%)$ were exhibiting poor conservation of energy. It was determined that the majority of the error was accumulated in a single integration step. This is perhaps not surprising as we are investigating fairly high energy systems undergoing abrupt changes in momentum. All simulations were performed using an in-house simulation code coupled to Mopac2012 [41].

\section{Results and Discussion}

We begin our analysis by examining the overall reactivity for the species, as shown in Figure 1. In this work, we define a trajectory to be "reactive" when one or more covalent bonds are broken and do not reform during the course of the simulation. At this collision energy, all collisions observed are impulsive in nature, with the peptide bouncing off of the surface, and we classify a trajectory as a Fast Fragmentation Event (FFE) if the first bond cleavage event occurred within 300 fs of the bounce, i.e. the turning point of the trajectory. All of the product ions that we observe are singly charged after they become separated from any other peptide fragments. There are three immediate trends that are observed on this time scale: 1) Reactivity decreases with peptide size, 2) FFE both decreases with system size, but also becomes relatively more important, and 3 ) the $\mathrm{A}_{\mathrm{n}} \mathrm{K}$ series (including
$\mathrm{AcA}_{7} \mathrm{~K}$ ) is more reactive than the $\mathrm{KA}_{\mathrm{n}}$ series (including $\mathrm{AcKA}_{7}$ ). There is an important realization to make regarding the fractions shown in Figure 1, namely that the fraction of FFE trajectories cannot change whereas the fraction of non-FFE trajectories depends on the overall simulation time. Hence, the observed difference between $\mathrm{AK}$ and KA in overall reactivity will not necessarily prove to hold in the long time limit as the dynamics of KA are likely slower due to the extra stabilization present. This trend continues throughout the $A_{n} K$ and $K_{n}$ series, with the latter having reduced overall reactivity due to the favorable energetics for that family of structures.

The FFE fraction is fixed and cannot change with a longer simulation time. It is interesting to note that the FFE fraction depends on the system size and decreases by more than a factor of four, moving from the smallest to the largest system size. At first, this observation may be surprising as one could argue that FFE should not strongly depend on size as it is the result of a strong local force, i.e. impulsive impact with the surface. We believe that the reason the system size clearly does have a large effect on the FFE fraction is that the energy transfer is distributed among more parts of the molecule as the ion's size increases. It is known that the percentage of translational to internal energy transfer does not depend strongly on system size within the range of system sizes and collision energy considered here $[33,35,36]$. Hence, roughly the same percentage of the $100 \mathrm{eV}$ collision energy is being put into, for example, both $\mathrm{AK}$ and $\mathrm{AcA}_{7} \mathrm{~K}$. However, the number of atoms - and therefore chemical groups - that come into close contact with the surface is dramatically different. With a greater number of groups accepting energy, there are more bonds within which to distribute the same amount of energy. This makes FFE less likely for a larger system. This also suggests that there could be a strong orientational effect on FFE, though we have not examined that here.

The question of the role of secondary structure is not conclusively answered from data in Figure 1. Although it is seen, for example, that $A_{5} \mathrm{~K}$ (alpha-helical) has a larger FFE fraction than $\mathrm{KA}_{5}$ (globular) the same is true for $\mathrm{A}_{3} \mathrm{~K}$ vs $\mathrm{KA}_{3}$, which are both globular in nature. Therefore, the primary sequence itself appears to be more important. However, this may also be an artifact of these two particular series of peptides, namely that $\mathrm{KA}_{n}$ naturally has more energetically favorable structures than $A_{n} K$. This would also be in line with the cluster-surface shattering work, which determined that the fragment distribution is related to the species stability [7].

To continue our examination of FFE, we will use the 
standard nomenclature scheme [19] as depicted in Figure 2. Our direct dynamics simulations automatically track connectivity. This information can be used to determine the backbone as well as sidechain cleavage sites. To ease the discussion, we will now turn our attention to three classes of FFE products: so called "simple FFE products" (which we define below), backbone rearrangements, and side chain fragmentation.

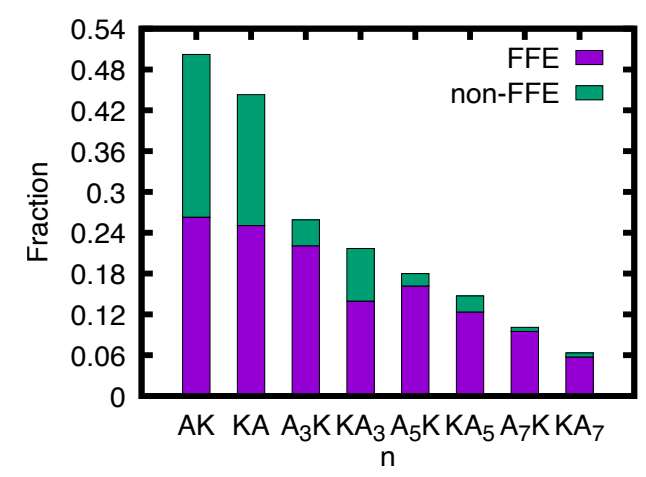

Figure 1: The fraction of reactive trajectories broken down into FFE and non-FFE as a function of species at the end of five picoseconds. The fraction is relative to the total number of trajectories. Note that $\mathrm{A}_{7} \mathrm{~K}$ and $\mathrm{KA}_{7}$ are in fact acetylated, and that additional fragmentation will occur outside the simulation timeframe. See Appendix A to see the conformation of each.

\subsection{Simple FFE Products}

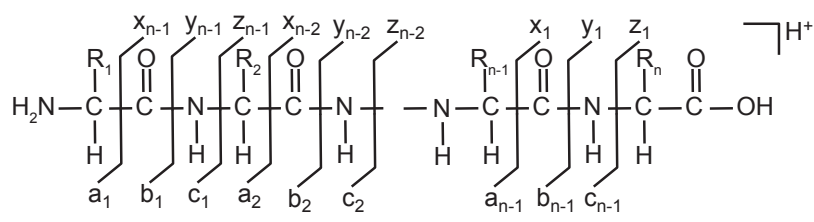

Figure 2: Definition of ion types. See [19] for greater detail.

Simple FFE products are those whose final ion contain a partially intact backbone that could have resulted from a single backbone cleavage event. This does not mean that the trajectory in fact only had a single backbone cleavage, but merely that the charged portion of the peptide could have resulted from a single backbone cleavage. For example, if the final product ion is an a1 ion, it is entirely possible for the b1 site to have also broken, resulting in the formation of $\mathrm{CO}$ as well as the remainder of the neutral peptide along with the a1 ion. Hence, the ion from a simple FFE product corresponds to the information directly provided by an experimental mass spectrum. Figure
3 examines the location of the backbone cleavage event as a function of ion type irrespective to location along the backbone, meaning both an $\mathrm{a}_{1}$ and $\mathrm{a}_{4}$ would both contribute to the fraction relative to all FFE events for the a-type fragmentation. It is important to note that this figure does not suggest that all of the ions generated would have the $m / z$ value associated with an a-ion because there could be other rearrangements that take place, such as a methyl shift. Rather this figure provides information regarding the most likely type of bond to break within the backbone along with which side of the peptide is charged following the bond cleavage. The fractions do not add up to 1 in this figure because not all FFE products are simple FFE products.

Figure 3 shows clear differences between the $A_{n} K$ and $\mathrm{KA}_{n}$ series. In the $\mathrm{KA}_{\mathrm{n}}$ series, the a-type ion is the dominant pathway for all species. In the $A_{n} K$ series, both the a- and x-type ions are common, with the x-type dominating at large system size and the a-type dominating at small system size. It is also possible to examine the most probable bond cleavage sites individually, which is shown in Figure 4. In this figure, the heavy atoms of each species are shown along with lines that designate the backbone cleavage location. Red lines denote that the charge is towards the N-terminus, whereas blue lines denote the charge is towards the $\mathrm{C}$-terminus. The thickness of the line provides the relative importance of that cleavage site. Figure 4 shows that although the a-type ion is dominant for the $\mathrm{KA}_{\mathrm{n}}$ series, the cleavage events are distributed among several possible a-sites. For $\mathrm{KA}_{n}$ with $\mathrm{n}=$ 1,3 and 5 , the a1 site is the most likely cleavage point. For example, in the $\mathrm{KA}_{3}$ system, the $\mathrm{a}_{1}$ site is nearly twice as likely as either the $\mathrm{a}_{2}$ or the $\mathrm{a}_{3}$ site. For $\mathrm{AcKA}_{7}, \mathrm{a}_{4}$ and $a_{7}$ sites are the most likely cleavage locations, with the $\mathrm{a}_{4}$ site being a little less than twice as likely than the $\mathrm{a}_{1}$ site. That said, there are a significantly smaller number of FFE events for this system (see Figure 1).

Seeing an a-type ion result from the $\mathrm{KA}_{\mathrm{n}}$ series is somewhat surprising, but it is indeed what is observed in our simulations. The ion forms through a multi-step mechanism. For example, an $\mathrm{a}_{1}$ ion could form through the following steps: 1) homolytic bond cleavage between the $\mathrm{C}_{\alpha}$ and $\mathrm{C}_{\beta}$ resulting in a $2+$ immonium-like ion and a 1- ion from the remainder of the peptide. Note here, that the two "ions" are extremely close to each other and calling them separate species at this point is an arbitrary decision to aid in the description of the mechanism. Full charge separation does not occur between these "ions". 2) proton transfer from the immonium-like ion returns the system to $1+$ ion and a neutral fragment, which do have 
full charge separation. The proton that transfers does not need to come from the nitrogen of the lysine, but rather can transfer from one of the carbon atoms of the backbone, which is why we refer to this ion as "immoniumlike". The entire process, from intact peptide, to forming both a $2+$ and 1- ion, back to an immonium-like 1+ ion and neutral fragment takes approximately 250 fs or less. If the proton transferred from a carbon atom rather than the lysine nitrogen, the immonium-like ion can rearrange, with time, to be the traditional immonium ion, which was also seen in some trajectories. Similar mechanistic steps occur for other a-type ions.

Turing our attention to the $A_{n} K$ series, Figure 4 highlights the shift in preference between a-type and b-type cleavage events with system size. The $\mathrm{a}_{1}$ site is the dominant cleavage location for $A K$, whereas the $a_{1}$ site is in competition with the $\mathrm{x}_{3}$ site as well as the $\mathrm{x}_{2}$ and $\mathrm{a}_{2}$ sites for $\mathrm{A}_{3} \mathrm{~K}$. Both $\mathrm{A}_{5} \mathrm{~K}$ and $\mathrm{AcA}_{7} \mathrm{~K}$ are alpha helical, and here, we see the $\mathrm{x}$-type cleavage events becoming dominant. Once again, we see a distribution of sites in the middle of the sequence with $\mathrm{x}_{4}$ and $\mathrm{x}_{5}$ being the most likely and roughly twice as likely as the $\mathrm{x}_{1}$ site. The $\mathrm{A}_{\mathrm{n}} \mathrm{K}$ series also retains some a-type cleavage events, with $\mathrm{a}_{4}$, for example, being a little less than half as likely as $\mathrm{x}_{4}$. This is a difference between the two series - $\mathrm{A}_{n} \mathrm{~K}$ has some competition between $\mathrm{x}$-type and a-type throughout whereas the $\mathrm{KA}_{\mathrm{n}}$ series is dominated by a-type.

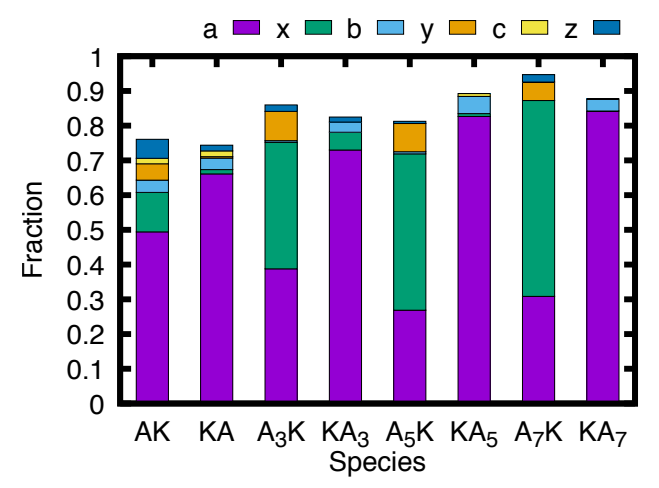

Figure 3: The fraction of ion sites for FFE fragmentation events that results from a single cleavage along the backbone. We note that we are using the ion nomenclature to identify the bond cleavage sites and the side of the peptide retaining the charge - not the final ion $\mathrm{m} / \mathrm{z}$, since additional rearrangements are possible.

\subsection{Backbone Rearrangements}

As described above, Figure 3 does not have fractions that add up to 1 because there are fragmentation events that are not simple FFE products. One way for this too occur is for an ion to require at least two backbone cleavage events. There are at least two ways for this to occur: Loss of both the $\mathrm{N}$ and $\mathrm{C}$-terminus, or 2) loss of a portion of the backbone between the $\mathrm{N}$ and $\mathrm{C}$-terminus followed by recombination. While both are observed in our simulations, neither are very likely. For example, for the $\mathrm{A}_{5} \mathrm{~K}$ peptide, which shows the lowest fraction of simple FFE events, there are 24 backbone rearrangement events, which as a fraction of the total number of FFE events is 0.15. 15 of these events result in a fragment that contains both the $\mathrm{N}$ and $\mathrm{C}$-terminus. There are at least five ways that we have observed for a portion of the middle of the backbone to be removed: 1) One of the simplest is the loss of the alpha carbon along with the $\mathrm{R}$ group of an alanine, namely the loss of $\mathrm{C}_{2} \mathrm{H}_{4}$. When this occurs the nitrogen of the amino acid binds to the carbonyl carbon. 2) It is also possible to lose the majority of an entire alanine amino acid. This typically occurs as $\mathrm{H}_{5} \mathrm{C}_{2} \mathrm{~N}+\mathrm{CO}$ loss. 3) A variation of the previous type of loss is for the $\mathrm{CO}$ to be retained in the backbone (loss of just $\mathrm{H}_{5} \mathrm{C}_{2} \mathrm{~N}$ ). When this occurs, the backbone contains a carbonyl carbon to carbonyl carbon bond, i.e. a $(\mathrm{O}=\mathrm{C})-(\mathrm{C}=\mathrm{O})$ sequence is formed. Loss of $\mathrm{H}_{5} \mathrm{C}_{2} \mathrm{~N}+\mathrm{CO}$ combined with loss of just $\mathrm{H}_{5} \mathrm{C}_{2} \mathrm{~N}$ is the most common backbone rearrangement observed for both $A_{n} K$ and $K A_{n}$. 4) A more exotic mechanism involves an exchange between the alpha and beta carbons, namely the nitrogen atom migrates from the alpha to beta carbon while the carbonyl group on the beta carbon shifts to the alpha carbon. This results in loss of $\mathrm{H}_{4} \mathrm{C}_{2} \mathrm{O}$. 5) The most complicated mechanism involves the cleavage of a peptide bond and the $\mathrm{C}_{\alpha}-\mathrm{C}_{\beta}$ bond further down the chain. These two cleavage sites recombine with the carbonyl carbon of the peptide bond binding to the oxygen of the carbonyl from the $\mathrm{C}_{\alpha}-\mathrm{C}_{\beta}$ cleavage event, and results in a backbone that contains a $\mathrm{C}-\mathrm{O}=\mathrm{C}$ sequence. Occurrence of this mechanism is rare, but highlights the complex nature of FFE reaction dynamics.

\subsection{Sidechain Fragmentation}

Another type of FFE event that is not captured in Figure 3 is fragmentation of the side chain alone. This type of event is even more rare than backbone rearrangements. Using $A_{5} \mathrm{~K}$ as an example, there are just 5 events that involve only side chain fragmentation, which is a 0.03 fraction of the total number of FFE events. Side chain fragmentation can also occur in combination with other bond cleavage events. This occurs through two major pathways: 1) loss of $\mathrm{NH}_{3}$ from the lysine, and 2) complete loss of the charged side chain. In the former, the system is stabilized by forming a cyclic structure, whereas in the 
<smiles>CC(C)(C#N)[C@H]1CO1</smiles>

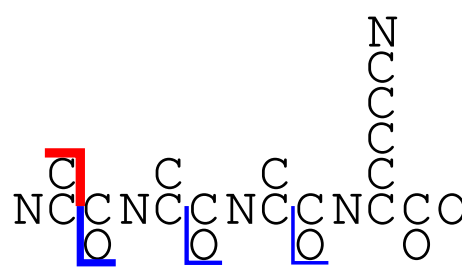

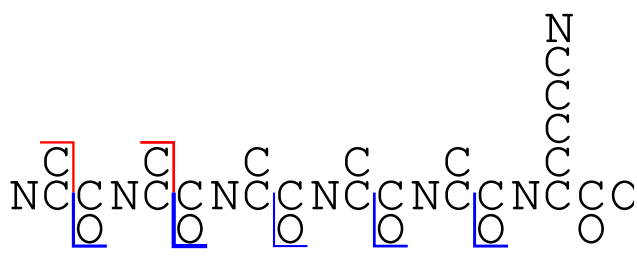
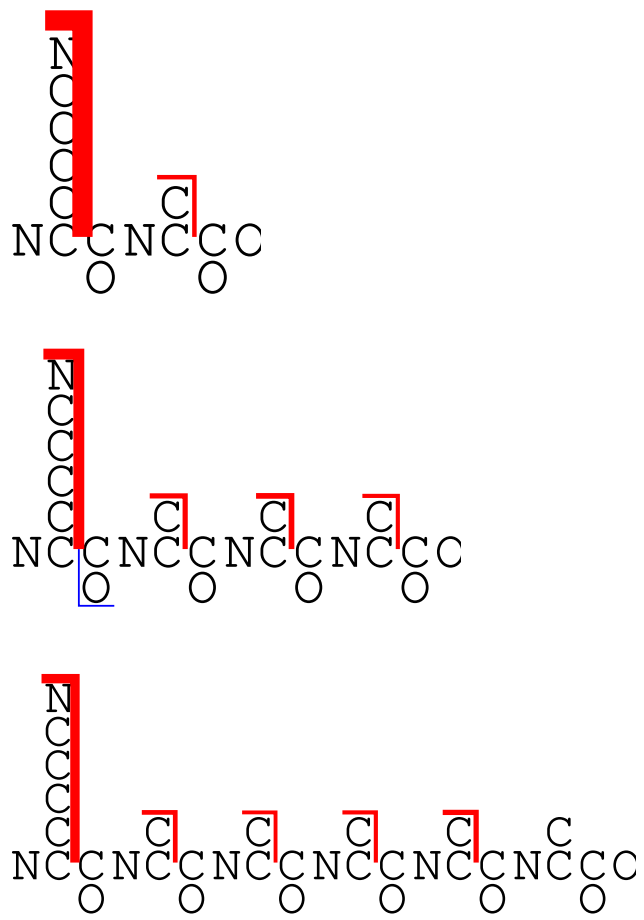

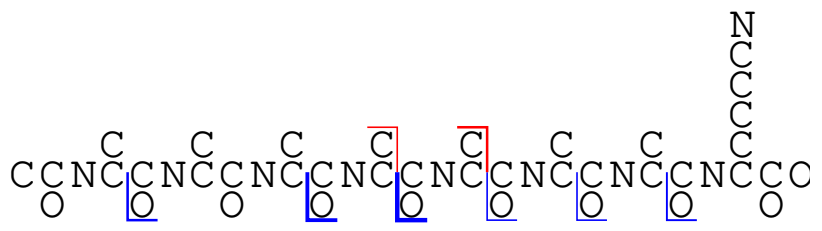

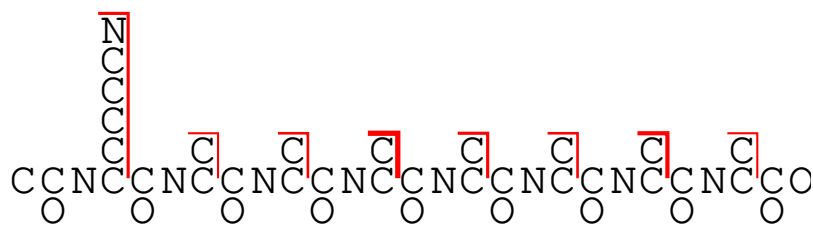

Figure 4: Depictions of the heavy atoms of each peptide overlaid with the location of the backbone fragmentation site. Fragmentation events in which the charge on the fragment containing the N-terminus are indicated by red lines, while those with the charge on the fragment containing the $\mathrm{C}$-terminus are blue. The thickness of the lines denotes the fraction of bond cleavage events that take place. Only cleavage events with a fraction of 0.05 relative to all FFE events are included for clarity. We note that backbone/side chain rearrangements can also occur and hence the mass of the final product may be different that that depicted in this representation.

latter the proton transfers back to the backbone.

\section{Summary}

Our simulation results show that there is a strong system size dependence on the fraction of FFE trajectories, with small systems having a greater likelihood of FFE than large systems for the same collision energy. This result is suggestive as to why few experiments have reported shattering products for protonated peptides, namely that most experimental systems are of sufficient size that it is plausible for shattering fragmentation to be a minor producer of final products.

Differences are also observed between the $A_{n} K$ and $\mathrm{KA}_{\mathrm{n}}$ family of peptides, with $\mathrm{A}_{\mathrm{n}} \mathrm{K}$ being more reactive than $\mathrm{KA}_{\mathrm{n}}$. However, $\mathrm{KA}_{\mathrm{n}}$ is more selective in the type of FFE fragmentation that is observed, with a-ions being prevalent for all system sizes. In contrast, $A_{n} K$ shows a preference for a-ions for small systems and $\mathrm{x}$-ions for large system sizes. We were not able to make conclusive statements regarding the importance of secondary structure. However, our data does support that either primary structure or overall energetics is an important driver of the FFE fraction.

The majority of FFE fragments, at the end of our short time simulations, can be described as simple FFE products meaning that the final charged product has a sequence of backbone atoms that could result from cleavage at a single backbone site. More complicated products are also observed that result from backbone rearrangements, although these are not as common as the simple products. Sidechain fragmentation alone is also possible, and in these systems is exclusive to the lysine amino acid group. 


\section{ACKNOWLEDGEMENTS}

GLB, AS, and MS gratefully acknowledge support from the National Science Foundation under grant number 1763652. GLB dedicates this work to Prof. William L. Hase, his post-doctoral advisor from 2008-2009, who passed away on Monday, March 23, 2020.

\section{Appendix A. Supplementary data}

Supplementary data to this article can be found online.

\section{References}

[1] A. Q. Stiving, Z. L. Vanaernum, F. Busch, S. R. Harvey, S. H. Sarni, V. H. Wysocki, Surface-Induced Dissociation: An Effective Method for Characterization of Protein Quaternary Structure (jan 2019). doi:10.1021/acs.analchem.8b05071.

[2] J. Laskin, Ion-surface collisions in mass spectrometry: Where analytical chemistry meets surface science, Int. J. Mass Spectrom. 377 (2015) 188-200. doi:10.1016/j.ijms.2014.07.004.

[3] V. H. Wysocki, K. E. Joyce, C. M. Jones, R. L. Beardsley, Surface-induced dissociation of small molecules, peptides, and non-covalent protein complexes., J. Am. Soc. Mass Spectrom. 19 (2) (2008) 190-208. doi:10.1016/j.jasms.2007.11.005.

[4] J. Laskin, T. H. Bailey, J. H. Futrell, Shattering of Peptide Ions on Self-Assembled Monolayer Surfaces., J. Am. Chem. Soc. 125 (6) (2003) 1625-1632. doi:10.1021/ja027915t.

[5] J. Laskin, J. H. Futrell, Energy Transfer in Collisions of Peptide Ions with Surfaces, J. Chem. Phys. 119 (6) (2003) 3413-3420. doi:10.1063/1.1589739.

[6] A. R. Dongré, J. L. Jones, Á. Somogyi, V. H. Wysocki, Influence of Peptide Composition, Gas-Phase Basicity, and Chemical Modification on Fragmentation Efficiency: Evidence for the Mobile Proton Model, J. Am. Chem. Soc. 118 (35) (1996) 83658374. doi:10.1021/ja9542193.

[7] T. Raz, R. D. Levine, On the shattering of clusters by surface impact heating, J. Chem. Phys. 105 (18) (1996) 8097-8102. doi:10.1063/1.472663.

[8] W. Christen, U. Even, Cluster impact chemistry, J. Phys. Chem. A 102 (47) (1998) 9420-9426. doi:10.1021/jp981874z.

[9] W. Christen, U. Even, T. Raz, R. D. Levine, Collisional energy loss in cluster surface impact: Experimental, model, and simulation studies of some relevant factors, J. Chem. Phys. 108 (24) (1998) 10262-10273. doi:10.1063/1.476487.

[10] D. G. Schultz, L. Hanley, Shattering of $\mathrm{SiMe}_{3}^{+}$during surfaceinduced dissociation, J. Chem. Phys. 109 (24) (1998) 10976. doi:10.1063/1.477737.

[11] O. Meroueh, W. L. Hase, Effect of Surface Stiffness on the Efficiency of Surface-Induced Dissociation, Phys. Chem. Chem. Phys. 3 (12) (2001) 2306-2314. doi:10.1039/b100892g.

[12] J. Laskin, J. H. Futrell, Surface-Induced Dissociation of Peptide Ions: Kinetics and Dynamics., J. Am. Soc. Mass Spectrom. 14 (12) (2003) 1340-1347. doi:10.1016/j.jasms.2003.08.004.

[13] Y. Wang, W. L. Hase, K. Song, Direct Dynamics Study of NProtonated Diglycine Surface-Induced Dissociation. Influence of Collision Energy., J. Am. Soc. Mass Spectrom. 14 (12) (2003) 1402-1412. doi:10.1016/j.jasms.2003.08.014.
[14] K. Park, K. Song, W. L. Hase, An ab initio direct dynamics simulation of protonated glycine surface-induced dissociation, Int. J. Mass Spectrom. 265 (2-3) (2007) 326-336. doi:10.1016/j.ijms.2007.03.009.

[15] K. Park, B. Deb, K. Song, W. L. Hase, Importance of Shattering Fragmentation in the Surface-Induced Dissociation of Protonated Octaglycine., J. Am. Soc. Mass Spectrom. 20 (6) (2009) 939-948. doi:10.1016/j.jasms.2009.02.028.

[16] V. H. Wysocki, G. Tsaprailis, L. L. Smith, L. A. Breci, Mobile and localized protons: a framework for understanding peptide dissociation., J. Mass Spectrom. 35 (12) (2000) 13991406. doi:10.1002/1096-9888(200012)35:12<1399::AIDJMS86>3.0.CO;2-R.

[17] R. Boyd, A. Somogyi, The mobile proton hypothesis in fragmentation of protonated peptides: a perspective., J. Am. Soc. Mass Spectrom. 21 (8) (2010) 1275-1278. doi:10.1016/j.jasms.2010.04.017.

[18] D. Frederickson, M. McDonough, G. L. Barnes, A Computational Comparison of Soft Landing of Alpha-Helical vs Globular Peptides, J. Phys. Chem. B 122 (41) (2018) 9549-9554. doi:10.1021/acs.jpcb.8b06232.

[19] I. A. Papayannopoulos, The interpretation of collision-induced dissociation tandem mass spectra of peptides, Mass Spectrom. Rev. 14 (1) (1995) 49-73. doi:10.1002/mas.1280140104.

[20] R. R. Hudgins, M. F. Jarrold, Helix Formation in Unsolvated Alanine-Based Peptides: Helical Monomers and Helical Dimers, J. Am. Chem. Soc. 121 (14) (1999) 3494-3501. doi:10.1021/ja983996a.

[21] K. Shaikh, J. Blackwood, G. L. Barnes, The Effect of Protonation Site and Conformation on Surface-Induced Dissociation in a Small, Lysine Containing Peptide, Chem. Phys. Lett. 637 (2015) 83-87. doi:10.1016/j.cplett.2015.07.062.

[22] M. D. Hanwell, D. E. Curtis, D. C. Lonie, T. Vandermeersch, E. Zurek, G. R. Hutchison, Avogadro: An Advanced Semantic Chemical Editor, Visualization, and Analysis Platform., J. Cheminform. 4 (1) (2012) 17. doi:10.1186/1758-2946-4-17.

[23] G. B. Rocha, R. O. Freire, A. M. Simas, J. J. P. Stewart, RM1: A Reparameterization of AM1 for H, C, N, O, P, S, F, $\mathrm{Cl}, \mathrm{Br}$, and I., J. Comput. Chem. 27 (10) (2006) 1101-1111. doi:10.1002/jcc.20425.

[24] G. L. Barnes, A. Podczerwinski, Simulating the Effect of Charge State on Reactive Landing of a Cyclic Tetrapeptide on Chemically Modified Alkylthiolate Self-Assembled Monolayer Surfaces, J. Phys. Chem. C 121 (27) (2017) 14628-14635. doi:10.1021/acs.jpcc.7b03478.

[25] Z. Homayoon, S. Pratihar, E. Dratz, R. Snider, R. Spezia, G. L. Barnes, V. Macaluso, A. Martin Somer, W. L. Hase, Model Simulations of the Thermal Dissociation of the TIK $\left(\mathrm{H}^{+}\right)_{2}$ Tripeptide: Mechanisms and Kinetic Parameters, J. Phys. Chem. A 120 (42) (2016) 8211-8227. doi:10.1021/acs.jpca.6b05884.

[26] Z. Gregg, W. Ijaz, S. Jannetti, G. L. Barnes, The Role of Proton Transfer in Surface-Induced Dissociation, J. Phys. Chem. C 118 (2014) 22149-22155. doi:10.1021/jp507069x.

[27] A. Geragotelis, G. L. Barnes, Surface Deposition Resulting from Collisions Between Diglycine and Chemically Modified Alkylthiolate Self-Assembled Monolayer Surfaces, J. Phys. Chem. C 117 (25) (2013) 13087-13093. doi:10.1021/jp402424z.

[28] W. Ijaz, Z. Gregg, G. L. Barnes, Complex Formation during SID and Its Effect on Proton Mobility, J. Phys. Chem. Lett. 4 (22) (2013) 3935-3939. doi:10.1021/jz402093q.

[29] G. L. Barnes, K. Young, L. Yang, W. L. Hase, Fragmentation and Reactivity in Collisions of Protonated Diglycine 
with Chemically Modified Perfluorinated Alkylthiolate-SelfAssembled Monolayer Surfaces., J. Chem. Phys. 134 (9) (2011) 094106. doi:10.1063/1.3558736.

[30] M. J. Abraham, T. Murtola, R. Schulz, S. Páll, J. C. Smith, B. Hess, E. Lindah, Gromacs: High performance molecular simulations through multi-level parallelism from laptops to supercomputers, SoftwareX 1-2 (2015) 19-25. doi:10.1016/j.softx.2015.06.001.

[31] J. P. Stewart, Mopac2016 http://openmopac.net (2016).

[32] A. Martin Somer, V. Macaluso, G. L. Barnes, L. Yang, S. Pratihar, K. Song, W. L. Hase, R. Spezia, Role of Chemical Dynamics Simulations in Mass Spectrometry Studies of CollisionInduced Dissociation and Collisions of Biological Ions with Organic Surfaces, J. Am. Soc. Mass Spectrom. 31 (1) (2020) 2-24. doi:10.1021/jasms.9b00062.

[33] S. Pratihar, G. L. Barnes, W. L. Hase, S. Pratihara, G. L. Barnes, W. L. Hase, Chemical Dynamics Simulations of Energy Transfer, Surface-Induced Dissociation, Soft-Landing, and Reactive- Landing in Collisions of Protonated Peptide Ions with Organic Surfaces, Chem. Soc. Rev. 45 (13) (2015) 3595-3608. doi:10.1039/C5CS00482A.

[34] S. Pratihar, G. L. Barnes, J. Laskin, W. L. Hase, Dynamics of Protonated Peptide Ion Collisions with Organic Surfaces. Consonance of Simulation and Experiment., J. Phys. Chem. Lett. 7 (2016) 3142-3150. doi:10.1021/acs.jpclett.6b00978.

[35] G. L. Barnes, W. L. Hase, Energy Transfer, Unfolding, and Fragmentation Dynamics in Collisions of N-Protonated Octaglycine with an H-SAM Surface., J. Am. Chem. Soc. 131 (47) (2009) 17185-17193. doi:10.1021/ja904925p.

[36] L. Yang, O. A. Mazyar, U. Lourderaj, J. Wang, M. T. Rodgers, E. Martinez-Núñez, S. V. Addepalli, W. L. Hase, Chemical Dynamics Simulations of Energy Transfer in Collisions of Protonated Peptide Ions with a Perfluorinated Alkylthiol SelfAssembled Monolayer Surface, J. Phys. Chem. C 112 (25) (2008) 9377-9386.

[37] O. Meroueh, W. L. Hase, Dynamics of Energy Transfer in Peptide-Surface Collisions, J. Am. Chem. Soc. 124 (7) (2002) 1524-1531. doi:10.1021/ja011987n.

[38] S. Pratihar, S. C. Kohale, S. A. Vázquez, W. L. Hase, S. A. Va, W. L. Hase, Intermolecular Potential for Binding of Protonated Peptide Ions with Perfluorinated Hydrocarbon Surfaces, J. Phys. Chem. B 118 (20) (2014) 5577-5588. doi:10.1021/jp410886s.

[39] S. Pratihar, N. Kim, S. C. Kohale, W. L. Hase, Mechanistic Details of Energy Transfer and Soft Landing in ala2-H+ Collisions with a F-SAM Surface, Phys. Chem. Chem. Phys. 17 (38) (2015) 24576-24586. doi:10.1039/C5CP03214H.

[40] C. Schlier, A. Seiter, High-Order Symplectic Integration: An Assessment, Comput. Phys. Commun. 130 (1-2) (2000) 176-189. doi:10.1016/S0010-4655(00)00011-4.

[41] J. P. Stewart, Mopac2013; http://openmopac.net (2012). 